\title{
Post-Depletion Aggression Restrained: Replicability of Brief Mindfulness Induction In Indonesian Sample
}

\author{
Cleoputri Yusainy ${ }^{1,2} \mathcal{E}$ Wahyu Wicaksono ${ }^{2}$ \\ ${ }^{1}$ Department of Psychology, Faculty of Social and Political Sciences, Universitas Brawijaya \\ ${ }^{2}$ Placebo Research Group
}

\begin{abstract}
Mindfulness practice is being promoted in Western countries as a means to improve one's ability to restrain aggression under "depleted" condition. The applicability of this framework in non-Western settings is yet to be determined. In this experiment $(N=$ 119 Indonesian undergraduates), we directly replicated Yusainy and Lawrence (2015) study with native British sample examining the effect of laboratory-induced mindfulness on postdepletion aggression (i.e., blast intensity in an adapted competitive reaction-time task). Similar results were obtained, in that mindfulness induction moderated the link between ego-depletion and (i) blast intensity under low/moderate provocation, and (ii) self-control performance after the aggression task. Notably, the benefit of mindfulness was also indicated in our additional aggression measure of the late deliverance of maximum blast in depleted females. While Western operationalization of mindfulness operates quite similarly across cultures, the inclusion of a subtle measure of aggression appears to be crucial for Indonesian females.
\end{abstract}

Keywords: aggressive behaviour; cross-cultural replication; ego-depletion; induction; mindfulness self-control

"One could say that there are three ways to get rid of anger: Kill the opponent, kill yourself, or kill the anger. Which one makes most sense to you?" (Allan Wallace). This quotation points out one's perplexity in dealing with angry feelings and refraining from aggressive responding. Some of us would retaliate against the provoking agent. Others could prefer taking the anger out on innocent others or on inanimate objects. A few might even deliberately hurt themselves, presumably to limit the accumulation of hostile thoughts towards the provocateurs (Yusainy \& Lawrence, 2014). While these acts could temporary make us feel better, they do not get the "anger fire" out of the system. In fact, venting anger retains the angry feelings active in the memory - similar to adding fuel to the flame (Bushman, 2002). This process is commonly followed by rumination, a repetitive and uncontrollable thoughts about one's own negative experiences (Denson, 2013).

Aggression in children, adults, and animals is dichotomised into "reactive aggression" encompassing defensive responses to situational triggers vs. "proactive aggression" in the form of deliberate actions being controlled by external reinforcements (Crick \& Dodge, 1996; see also Baron \& Richardson, 1994;

\footnotetext{
${ }^{1}$ Address for corespondence:

cleo.yusainy@ub.ac.id
} 
Berkowitz, 1993; Geen, 2001). Although these two types of aggression can be combined in the same action, their neural pathways are different, thus supporting the nature and evolution of aggression (Bartholow, 2018; Wrangman, 2017). The current study focuses on reactive aggression, since this type of aggression is generally more sensitive to interventions (McEllistrem, 2004). Specifically, we measure reactive aggression when the physical harm on a target is delivered faceto-face or where the perpetrator can be identified (i.e., direct physical aggression: Björkqvist, Lagerspetz, \& Kaukianen, 1992).

The ultimate goals of aggression may vary but the immediate intention to harm others who are motivated to avoid the aggressive actions is critical as a proximate goal (Anderson \& Bushman, 2002). Aggression occurs when a combination of personal and situational factors triggers angry feelings, hostile thoughts, and arousal levels which influence subsequent appraisal and decision processes (Allen, Anderson, \& Bushman, 2018). Consequently, the impact of an aggressiontriggering situation (e.g., provocation) could be exaggerated or undermined by one's ability to alter, override, or manipulate aggression-related feelings and thoughts. Indeed, the inability to control oneself is a leading factor in criminality and violence (Gottfredson \& Hirschi, 1990). Lower level of self-control is also associated with difficulty in identifying and describing one's own feelings (Yusainy, 2017). In contrast, rates of behavioural problems and criminality over life-course development are lower amongst selfcontrolled individuals (Caspi, 2000; Moffitt et al., 2011). Exerting good self-control, however, requires sacrifice.

The prominent strength model (Baumeister, Bratslavsky, Muraven, \& Tice,
1998; Baumeister, Heatherton, \& Tice, 1994) views self-control as a common resource that becomes depleted with use (see Baumeister, Vohs, \& Tice, 2007). This "egodepletion" effect restricts initiation of selfcontrol acts in the attentional neural system (Inzlich \& Gutsell, 2007), thereby making the self temporary incapable of performing further, seemingly unrelated self-control acts. Various domains of self-control have been investigated within the sequentialtask paradigm to suggest evidence for the strength model proposition (see Hagger, Wood, Chris, \& Chatzisarantis, 2010).

Supports for the strength model in the aggression literature is provided through many aggression paradigms, ranging from negative evaluation task (Stucke \& Baumeister, 2006), competitive reactiontime task (Vohs, Glass, Maddox, \& Markman, 2011), uncomfortable pose task towards intimate partner (Finkel, DeWall, Slotter, Oaten, \& Foshee, 2009), and inappropriate use of force by police officers (Staller, Christiansen, Zaiser, Körner, \& Cole, 2017). In the aforementioned studies, higher levels of aggression were found amongst depleted participants. It appears that a temporary failure of self-control is the proximal antecedent of aggression (Denson, DeWall, \& Finkel, 2012).

When self-control resource is at risk, mindfulness practices could increase sensitivity to the "in-the-moment" experiences signalling the need for control (Teper, Segal, \& Inzlicht, 2013). Mindfulness-based interventions are now being integrated as a part of the "thirdwave" cognitive-behavioural approaches for aggression in Western countries (Howells, Tennant, Day, \& Elmer, 2010; Ireland \& Batool, 2018; Shonin, Gordon, Slade, \& Griffiths, 2013). Mindfulness can be seen a mode of awareness compromising regulatory attention in the manner of 
curiosity, openness, and acceptance (Bishop et al., 2004). This definition emphasises the state-like quality of mindfulness.

Preliminary evidence for the moderation of laboratory induced mindfulness on post-depletion aggression was shown by Yusainy and Lawrence (2015). In their study, native British undergraduates who performed an attention control task (as the depleting task) followed by a mindfulness induction task delivered less intense noise to opponents in an adapted competitive reaction-time task (CRTT: Taylor, 1967). Their experiment fills the gap in the literature for the immediate impact of mindfulness on aggression after depletion in the absence of extensive mindfulness training. They also found that mindfulness amplified performance on a subsequent self-control measure. The present study aims to replicate Yusainy and Lawrence framework in an Eastern culture sample, specifically Indonesia.

As a highly collectivist culture, there is a preference in Indonesia towards the Javanese value of prohibiting rude conduct, shouting, or open conflict (Koentjaraningrat, 1985). Conflict resolution through direct communication is seen as unacceptable since it could endanger relationships and group harmony (Hofstede, Hofstede, \& Minkov, 2010; see Hofstede's insights https://www. hofstede-insights.com/country-

comparison/indonesia/). Cross-cultural studies support that compared to those from individualistic cultures, Indonesian children less likely displayed direct aggressive acts (Bergeron \& Schneider, 2005; Bergmüller, 2013; French, Jansen, \& Pidada, 2002).

Characteristics of mature Javanese individuals include the effort to maintain internal and external harmony as well as the existence of awareness and control (Trimulyaningsih, 2017). As members of cultures that promote harmonious interdependence typically engage in daily selfcontrolled interaction (Markus \& Kitayama, 1991), they may become better at self-control and less prone to ego-depletion (Seeley \& Gardner, 2003). In line with this proposal, our recent cross-country research ( $k=23$, total $N=2,141)$ directly replicated the standardised sequential-task paradigm and found that for the Indonesian sample $(n=156)$, the size of ego-depletion effect on task performance was relatively small (see Hagger et al., 2016). Whether this insignificant ego-depletion effect also occurs in the context of a more complex experimental manipulations (i.e., involving both measures of aggressive behaviour and performance in self-control) is yet unknown.

More crucially, the concept of mindfulness originates from Eastern contemplative tradition of remembering to pay attention to and be aware of the present moment (Wallace \& Bodhi, 2006). Given that most mindfulness studies are conducted using Western populations (Van Dam et al., 2018), it is necessary to test its efficacy on post-depletion aggression beyond the Western sample. Direct replication is necessary to provide some evidence on the applicability of this framework with a different population of participants (Yusainy, 2015).

As a direct replication of Yusainy and Lawrence (2015) research, the current study employs their version of the CRTT as a method of aggression. The CRTT is one of the most popular laboratory aggression paradigms (McCarthy \& Elson, 2018). In this computer-based reaction-time task, participants are allowed to deliver a blast of noise to an opponent each time they win a trial. Direct physical aggression is 
measured by the intensity of participants' noise blasts (Elson, Mohseni, Breuer, Scharkow, \& Quandt, 2014; Giancola \& Parrot, 2008).

To our knowledge, this is the first study to examine the CRTT in Indonesian sample. To demonstrate some validity for the paradigm, the blast intensity delivered by participants should increase with additional provocation (i.e., blast intensity received by participant across levels of provocation). Also, as in the previous British sample, male participants here should deliver higher levels of blast intensity under conditions of no and low/moderate provocation (for sex differences in aggression, see e.g., Archer, 2004; Bettencourt \& Miller, 1996; Giancola \& Parrot, 2008).

Notably, an additional aggression measure in the form the delay duration before the maximum blast was delivered the opponent in the CRTT (i.e., maximum latency; see Lawrence \& Hutchinson, 2014) is included in the current study. Giancola and Parrot (2008) suggested that when factors such as cultural values and sex role norm inhibit aggressive impulses, implicit aggression (shock duration in the CRTT) is more likely than explicit aggression (shock intensity). The subtleness of shock duration is arguably similar to maximum blast latency. In this way, the moderation of mindfulness induction can be explored on the link between ego-depletion and different forms of aggression.

Following the original study, we incorporate a second measure of performance in self-control, specifically physical stamina (i.e., a handgrip task). Squeezing a handgrip has been identified as one of the frequently used dependent tasks in the sequential-task paradigm (Hagger et al., 2010). While depleted participants' duration of squeezing the handgrip should decrease relative to baseline, we also expect this effect to be less evident amongst those who then receive mindfulness induction.

Altogether, we predict that the benefits of mindfulness found in Yusainy and Lawrence (2015) study with British participants (i.e., reductions in postdepletion blast intensity and improvement in physical stamina) may occur in our sample, with an addition of reductions maximum blast latency in the CRTT. However it is also plausible for the pattern of findings to differ from the original study, given that sample in Indonesia may not be familiar with mindfulness procedures derived from Western conceptualisation.

\section{Methods}

\section{Participants and procedures}

Our study was approved by local ethics committee. We directly replicated the design and protocols from Yusainy and Lawrence (2015; see Fig. 1). G*Power 3.1. calculated a sample size of 128 for medium effect $(d=.25)$ from a 2 (ego-depletion: depletion vs. no depletion] $X \quad 2$ (mindfulness induction: mindfulness induction vs. no mindfulness induction) condition and 1 covariate (participant's sex) at the power of .80 and an alpha level of .05 (http://www.gpower.hhu.de/). With reference to the original study, a sample size of 110 is sufficient. Our study was able to recruit 124 undergraduates from a large university in East Java, Indonesia. These participants were assigned randomly based on sex to one of the four experimental conditions. Two participants fell asleep during the mindfulness induction task and three of them expressed spontaneous suspicions to the CRTT, resulting in 119 final participants $(60$ females; mean age $=$ 
$20.40, S D=1.24)$. None of them had recently encountered formal mindfulness practices.

As in the original study, we recruited potential participants via posters/leaflets on campus for a study aimed to examine the way people perform in a competitive reaction-time task. They were given small amounts of inconvenient allowance and a chance to win an incentive of Rp 150.000,00 for the fastest participants' reaction-time. The rest of the procedure followed Yusainy and Lawrence (2015) study.

\section{Materials and apparatus}

Two postgraduate Indonesian students translated the self-reported measures and experimental protocols from the original study, and a commercial translation service back-translated the materials into English and checked against the original transcript. We used two pilot participants for pretesting these adapted measures.

For the depletion task (i.e., attention control), we told participants that they would be making judgments (measured by three dummy questions) about a local woman being interviewed off-camera. While the 6-mins video of interview was presented, a series of common one-syllable words appeared at the bottom of the screen ( $n=36$ words; $10 \mathrm{~s}$ each). Some words were translated directly in accordance to their meaning in the original study (e.g., "glue" Indonesian: lem; "tire" - ban). Other words could not be translated into one-syllable words (e.g., "ten" - sepuluh; "book" - buku; "shoe" - sepatu) so they were replaced with comparable Indonesian words (e.g., nol; map; sol). Instructions of to not read or look at these words were given to participants in the depletion condition only. The two questions measuring depletion condition (i.e., difficulty and effort) were positively correlated $(r=.46, p<.0001)$.
A translated audio instruction from the "mindfulness of body and breath" (Williams \& Penman, 2011) was given to participants in the mindfulness induction condition. The no mindfulness induction participants listened to two neutral educational excerpts and then arranged spontaneous words from a scrabble set. The task for the no mindfulness induction condition could be considered an active "neutral" task because participants may compose the letters in any possible ways they could think of without obeying typical scrabble game rules. Arguably, this type of task does not include uncontrollable and social-evaluative elements related to stress (e.g., Dickerson \& Kemeny, 2004), but still requires comparable amount of concentration to that in the mindfulness condition. Both manipulation lasted for 15 minutes. We obtained adequate reliability for the state measure of mindfulness (Toronto Mindfulness Scale (TMS): Lau et al., 2006) in terms of curiosity ( $\alpha=.76 ; 6$ items) and decentering ( $\alpha=.62 ; 7$ items). Curiosity and decentering were positively correlated $(r=.61, p<.0001)$.

The aggression task of adapted CRTT was presented using E-prime software as a series of reaction-time trials, in which participants have to hit the spacebar on the computer keyboard when a white circle stimuli appeared on the screen. Winner of each trial could select a level ranging from 0 (no blast) through to 8 (maximum blast). Participant experienced no provocation (first win trial), low/moderate provocation (blast levels 1-4; 40 trials), and high provocation (blast levels 5-8; 40 trials) from a bogus opponent. In addition to blast intensity, the current study also recorded the maximum blast latency (i.e., the number of trials participants waited before delivering the maximum blast) in the CRTT. 
For the second measure of self-control in physical stamina domain, a manual hand exerciser was used as a handgrip stamina task. We asked participants to squeeze the handgrip with their dominant hand continuously until the wad of paper in between the handles fell out. The task was given (i) at baseline, (ii) pre-CRTT, and (iii) post CRTT. Handgrip stamina was calculated by subtracting the baseline duration from the subsequent durations (Hagger et al., 2010). Handgrip stamina changes pre- and post-CRTT were positively correlated $(r=.62, p<.0001)$.

As in the original study, we incorporated measure of post-depletion and post-aggression mood (Positive Affect, Negative Affect Schedule (PANAS): Watson, Clark, \& Tellegen, 1988). The reliability in the current sample was good for post-depletion mood (PANAS1: $\alpha=.79$; 10 items and .81; 10 items for PA1 and NA2) and post-CRTT mood (PANAS2: $\alpha=.84$ for both PA2 and NA2). The association between ego-depletion and mood is typically predicted as null (Baumeister et al., 1998). Provided the aversive nature of the depleting task, however, mood particularly negative affect can also relate to ego-depletion (Hagger et al., 2010). Likewise, the role of negative affect on aggression has been well-documented (Anderson \& Bushman, 2002; Berkowitz, 1993).

\section{Plan of analyses}

We carried out the same regression path analysis modeling from the replicated study to test the role of mindfulness induction in moderating the effect of depletion on aggression. Specifically, we used Hayes's PROCESS macro for simple moderation (http://www.processmacro. org/index.html; see Hayes, 2018) for testing the moderation effect of mindfulness induction on the depletion and blast intensity link on each provocation level, i.e., no provocation, low/moderate provocation, high provocation. Blast intensity was expected to be predicted by depletion condition (Hypothesis 1a) and the interaction between depletion and mindfulness induction (Hypothesis 1b), particularly under low/moderate provocation. Similar analyses were conducted to examine the effect of mindfulness induction on the depletion and self-control performance (i.e., handgrip stamina) link, in terms of changes in handgrip duration pre-CRTT and postCRTT relative to baseline. We expected that compared to no ego-depletion participants, depletion participants would be less capable to maintain their baseline level (Hypothesis 2a), but this effect would be moderated by mindfulness induction (Hypothesis 2b). Hypotheses $1 \mathrm{~b}$ and $2 \mathrm{~b}$ would be supported if the interaction coefficient between depletion and mindfulness induction is statistically different from zero.

For the additional aggression measure of the maximum blast latency (i.e., the number of trials participants waited before delivering the maximum blast), we conducted a censored survival analysis using Cox regressions. We expected the maximum blast latency to be predicted by ego-depletion (Hypothesis 3a) and the interaction between depletion and mindfulness induction (Hypothesis $3 b$ ). 


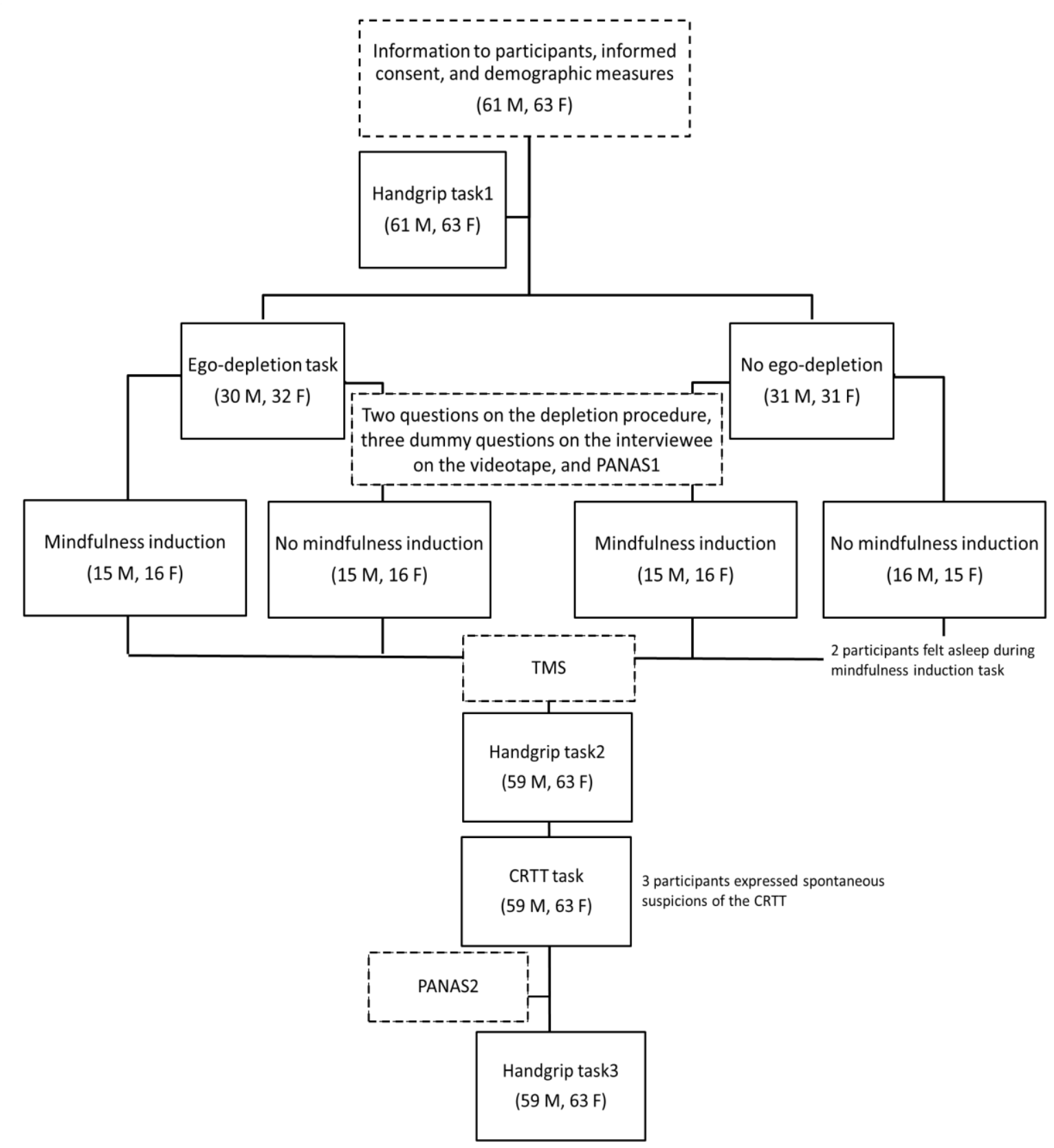

Figure 1. Flow of participants. Note. PANAS: Positive Affect, Negative Affect Schedule (PANAS: Watson et al., 1988); TMS; Toronto Mindfulness Scale (Lau et al., 2006); CRTT: Adapted Taylor competitive reaction-time task (Yusainy \& Lawrence, 2015). M = Males; F = Females

\section{Results}

\section{Manipulation check}

The ego-depletion (attention control) manipulation was effective. Participants in the depletion condition $(n=59)$ rated the task as more difficult than those in the no depletion condition $(n=60 ; t(117)=4.35, p<$
$.0001 ; M$ depletion $=3.68, S D=1.73$ vs. $M$ no depletion $=2.37, S D=1.56$ ) and having controlled their attention to a greater extent than no depletion participants $(t(117)=4.65$, $p<.0001 ; M$ depletion $=3.78, S D=1.77$ vs. $M$ no depletion $=2.33, S D=1.62$ ).

The mindfulness induction task succeeded in increasing participants decentering $(t(117)=2.36, p<.05 ; M$ 
mindfulness induction $=2.38, S D=.47$ vs. $M$ no mindfulness induction $=2.18, S D=$. $47)$, but not their curiosity $(t(117)=1.65, p=$ .10; $M$ mindfulness induction $=2.77, S D=$ .54 vs. $M$ no mindfulness induction $=2.61$, $S D=.53)$.

As predicted in the strength model, mood after the depletion task was equal between depletion and non-depletion conditions (PA1: $t(101.65)=-.75, p=.45$; NA1: $t(117)=.20, p=.85$; all $n s$.). Similarly, mood following the CRTT did not differ between depleted and non-depleted participants (PA2: $F(3,115)=1.57, p=.20$; NA2: $F(3,115)=1.05, p=.37$; all $n s$.$) . It$ should be noted, however, that although the mood measures were not related to most of the outcomes ( $p$ values ranged from .06 to $.98, n s$.), positive affect was related to blast intensity under high provocation (PA1: $r=.28, p=.01$; PA2: $r=.20, p=.03$ ). Accordingly, PA1 and PA2 would be included as covariates in the relevant moderation analysis.

Moderation of mindfulness induction on depletion and levels of blast intensity link

Table 1 presents the mean in blast intensity and change in handgrip stamina for the four experimental conditions in the current study. For blast intensity, a one-way repeated-measure ANOVA was performed to test differences in blast intensity under no provocation, low provocation, and high provocation trials. Unusually, blast intensity was not affected by level of provocation $(F(1.56,183.69)=1.88, p=.17)$. Participants did not deliver higher blast intensity under low provocation $(M=5.32$, $S D=1.78)$ compared to under no provocation $(M=5.61, S D=2.20 ; p=.06)$, or under high provocation $(M=5.37, S D=$ 1.94) compared to under low provocation $(p$ =.63). Nevertheless, the typical sex differences in the CRTT occurred in this sample, in that males delivered higher intensity blasts under conditions of no provocation $(t(117)=2.09, p<.05 ; M$ males $=6.03, S D=2.05$ vs. $M$ females $=5.20, S D=$ 2.30) and low provocation $(t(117)=2.36, p<$ $.05 ; M$ males $=5.70, S D=1.83$ vs. $M$ females $=4.94, S D=1.67)$, but not under high provocation compared to females $(t(109.28)$ $=.65, p=.52 ; M$ males $=5.49, S D=2.17$ vs. $M$ females $=5.26, S D=1.68)$. Participants' sex would also be included as covariate in the relevant moderation analysis.

Table 1.

Blast intensity across provocation levels and overall change in handgrip stamina

\begin{tabular}{llllllllllll}
\hline & \multicolumn{4}{c}{ Depletion } & \multicolumn{4}{c}{ No depletion } \\
\cline { 2 - 11 } & \multicolumn{1}{c}{$(1 \mathrm{a})$} & $(1 \mathrm{~b})$ & $(1 \mathrm{c})$ & $(2 \mathrm{a})$ & $(2 \mathrm{~b})$ & $(1 \mathrm{a})$ & $(1 \mathrm{~b})$ & $(1 \mathrm{c})$ & $(2 \mathrm{a})$ & $(2 \mathrm{~b})$ \\
\hline Mindfulness & 5.20 & 5.14 & 5.17 & 6.87 & -7.30 & 5.53 & 5.14 & 5.26 & 7.03 & -1.07 \\
induction, $M(S D)$ & $(2.43)$ & $(1.89)$ & $(2.00)$ & $(18.05)$ & $(12.42)$ & $(2.74)$ & $(2.17)$ & $(2.25)$ & $(18.75)$ & $(14.63)$ \\
No mindfulness & 6.34 & 6.14 & 6.11 & -15.76 & -18.65 & 5.40 & 4.86 & 4.98 & -3.87 & -9.07 \\
induction, $M(S D)$ & $(1.42)$ & $(1.17)$ & $(1.58)$ & $(14.99)$ & $(18.27)$ & $(1.94)$ & $(1.53)$ & $(1.72)$ & $(8.77)$ & $(15.28)$ \\
\hline
\end{tabular}

Note. (1) Blast intensity under (a) no provocation (b) low/moderate provocation (c) high provocation

(2) Changes in mean time that participants squeezed the handgrip (a) pre-CRTT (b) postCRTT. Higher positive scores indicate greater aggression and better handgrip stamina (selfcontrol performance).

Results for the moderation model of mindfulness induction on the link between depletion and blast intensity are presented in Table 2. Supporting Hypothesis 1a, there was a significant main effect of depletion on blast intensity under low/ moderate 
provocation $(p=.04)$. Mindfulness induction significantly moderated the link between depletion and blast intensity under low/moderate provocation only (Hypothesis 1b; $p=.04$ ). Specifically, following low/moderate provocation, participants in depletion condition who received mindfulness induction delivered lower levels of blast intensity compared to their counterparts without induction, whereas no differences were found between non-depleted participants with or without mindfulness induction.

The moderation analysis was then repeated while for controlling the hypothesised covariates (PA1 and PA2 for blast intensity under high provocation, and participants' sex for blast intensity under no and low/moderate provocation). A similar pattern of results was obtained, except under high provocation.
Specifically, by controlling positive affect after the depletion and CRTT, the previously non-significant interaction between mindfulness induction and depletion on blast intensity became significant $(B=-1.41, S E=.69, p=.04)$ with the total variance due the interaction increased from $2.52 \%$ to $3.29 \%$ when provocation from the opponent was high. However there was an absence of the usual main effect of depletion $(B$ increased from $=$ .52 to $.60, p=.08, n s$. for the main effect of depletion) and mindfulness induction $(B$ increased from $=-.33$ to $-.45, p=.19$ ). Either PA1 or PA2 predicted blast intensity under high provocation $(p s>.182, n s$.). The role of participants sex was more clear, in that being males increased the likelihood of higher levels of blast intensity under no provocation $(p=.03)$ and low/moderate provocation $(p=.01)$.

Table 2 .

Moderation model of mindfulness induction on the link between depletion and blast intensity/handgrip stamina

\begin{tabular}{|c|c|c|c|c|c|}
\hline \multirow{3}{*}{ Model tested } & \multicolumn{3}{|c|}{ Blast intensity } & \multicolumn{2}{|c|}{ Handgrip stamina } \\
\hline & $(1 \mathrm{a})$ & $(1 b)$ & (1c) & $(2 a)$ & $(2 b)$ \\
\hline & $B(S E)$ & $B(S E)$ & $B(S E)$ & $B(S E)$ & $B(S E)$ \\
\hline Depletion & $.30(.40)$ & $.63^{*}(.32)$ & $\begin{array}{c}.52 \\
(.35)\end{array}$ & $\begin{array}{l}-5.98^{*} \\
(2.87)\end{array}$ & $\begin{array}{l}-7.90^{* *} \\
(2.80)\end{array}$ \\
\hline Mindfulness induction & $-.50(.04)$ & $-.35(.32)$ & $\begin{array}{l}-.33 \\
(.35)\end{array}$ & $\begin{array}{c}16.71^{* * *} \\
(2.87)\end{array}$ & $\begin{array}{c}9.66^{* * *} \\
(2.80)\end{array}$ \\
\hline Depletion $x$ mindfulness & $-1.28(.80)$ & $-1.28^{*}(.63)$ & $\begin{array}{l}-1.23 \\
(.70)\end{array}$ & $\begin{array}{l}11.73^{*} \\
(5.74)\end{array}$ & $\begin{array}{c}3.35 \\
(5.60)\end{array}$ \\
\hline$R^{2}$ on interaction $(\%)$ & $2.11 \%$ & $3.25 \% *$ & $2.52 \%$ & $2.65^{* \%}$ & $0.03 \%$ \\
\hline Conditional effect of depletion without mindfulness & - & $1.29^{* *}(.45)$ & - & $\begin{array}{c}-11.89^{* *} \\
(4.07)\end{array}$ & - \\
\hline Conditional effect of depletion with mindfulness & - & $-.001(.44)$ & - & $.50(4.04)$ & - \\
\hline $\begin{array}{l}\text { ote: (1) Blast intensity under (a) no provocation }(b) \text { low } \\
\text { in mean time that participants squeezed the har } \\
\text { Bootstrap sample in the moderation models was } \\
\text { replacement }(95 \% \text { bias corrected confidence int } \\
\text { Coding: } 1=\text { depletion or mindfulness induction } \\
\text { condition. All predictors were mean-centered. } \\
B=\text { unstandardised regression coefficient; } S E= \\
{ }^{*} p<.05^{* *} p<.01^{* * *} p<.001\end{array}$ & $\begin{array}{l}\text { moderate } \\
\text { dgrip (a) pr } \\
\text { based on th } \\
\text { rvals). } \\
\text { ondition; } 0 \\
\text { tandard err }\end{array}$ & $\begin{array}{l}\text { rovocation (c } \\
\text { e-CRTT (b) p } \\
\text { e recommenc } \\
\text { = non-depleti } \\
\text { or; } R^{2}=\text { varia }\end{array}$ & $\begin{array}{l}\text { high pr } \\
\text { st-CRT } \\
\text { d size c } \\
\text { or no } \\
\text { ce incre }\end{array}$ & 5,000 resa & $\begin{array}{l}\text { 2) Changes } \\
\text { mples with } \\
\text { s induction }\end{array}$ \\
\hline
\end{tabular}


Moderation of mindfulness induction on depletion and handgrip stamina link

Handgrip stamina was calculated as a change scores, by subtracting the baseline duration from the subsequent durations (see again Table 1). No differences in handgrip stamina were found between sexes prior to the CRTT $(t(117)=-1.42, p=$ $.16, n s . ; M$ males $=-3.68, S D=19.23$ vs. $M$ females $=1.01, S D=16.68$ ). Female participants, however, were more able to return to their baseline duration after the CRTT $(t(117)=-2.16, p=.01 ; M$ males $=-12.80, S D$ $=17.19$ vs. $M$ females $=-5.15, S D=14.61$ ).

Results for the moderation model of mindfulness induction on the link between depletion and handgrip stamina (Table 2) revealed that the standard main effect of depletion on handgrip performance (Hypothesis 2a) was found pre-CRTT ( $p=$ $.04)$ and post-CRTT $(p=.02)$. The effect of mindfulness induction on post-depletion handgrip stamina (Hypothesis 2b) was partially supported, in that depleted participants who received mindfulness induction outperformed depleted ones with no induction pre-CRTT $(p=.04)$, but this moderating effect did not persist postCRTT ( $p=.40, n s$.$) . After the CRTT, non-$ depleted participants and those who received mindfulness induction performed better in the handgrip task.

A consistent pattern of results was obtained when we repeated the moderation analysis with participants' sex as covariate. Sex differences in handgrip stamina were insignificant pre-CRTT ( $p=.09$, ns.), but females outperformed males post-CRTT ( $p$ $=.005)$.

Moderation of mindfulness induction on the depletion and maximum blast latency link

Amongst those who had delivered the maximum blast $(N=98 ; 82.35 \%$ of total participants), the maximum blast latency was associated with $\operatorname{sex}(t(82.45)=2.46, p=$ $.02)$, such that females $(n=45 ; 71.43 \%$ of total females) waited significantly longer than males ( $n=53 ; 89.83 \%$ of total males) before delivering the maximum blasts $(M$ males $=7.11, S D=10.08$ vs. $M$ females $=$ 12.96, $S D=12.95$ ). Censored survival analysis using Cox regressions revealed that our proposed model (depletion $\mathrm{x}$ mindfulness induction on maximum blast latency) was not significant $\left(\Delta \chi^{2}=5.09, p=\right.$ $.17, n s$,$) .$

When we repeated the analysis controlling for participant's sex, the final model became marginally significant $\left(\Delta \chi^{2}=\right.$ 9.31, $p=.054)$. The point at which participants delivered the maximum blast was influenced by the interaction of mindfulness and depletion (Hypothesis 3b: Wald $=4.03, p=.045$, CI $[.19, .98], \exp (B)=$ .43 ), and sex (Wald $=4.23, p=.04$, CI [.42 to $.98], \exp (B)=.64)$. Specifically, depleted participants with mindfulness induction, and females were less likely to deliver maximum blast earlier to the opponent. It should be noted, however, that neither the main effect of depletion (Hypothesis 3a: Wald $=2.56, p=.20, n s .$, CI [.90, 2.82], $\exp (B)$ $=1.59$ ) nor of mindfulness induction (Wald $=.40, p=.52, n s ., \mathrm{CI}[.69,2.10], \exp (B)=1.20)$ was shown.

Given the importance of participant's sex in predicting the results, we repeated the censored survival analyses separately by sex. A different effect of depletion and mindfulness induction on maximum blast latency occurred. For females, the final model was significant $\left(\Delta \chi^{2}=8.88, p=.03\right)$. Depleted females who received mindfulness induction were less likely to deliver the maximum blast earlier (Wald $=5.77, p=$ .02 , CI [.06, .75], $\exp (B)=.21)$. There was also a significant main effect of mindfulness induction, in which females with 
mindfulness induction tended to give the maximum blast later (Wald $=7.86, p<.01$, CI $[1.45,8.20], \exp (B)=3.45)$, but no effect of depletion (Wald $=2.62, p=.11$, ns., CI [.86, $4.85], \exp (B)=2.04)$ on maximum blast latency. For males, the final model was not significant $\left(\Delta \chi^{2}=6.48, p=.09\right)$.

\section{Discussion}

The current study is a cross-cultural replication of Yusainy and Lawrence (2015) experiment using Indonesian university students. Before discussing the effect of experimental manipulations on aggressive behaviour, we should acknowledge that the absence of correlation between levels of provocation and blast intensity in the current sample is quite unusual in the CRTT experiments (see e.g., Anderson, Buckley, \& Carnagey, 2008; Lawrence \& Hutchinson, 2014; Yusainy \& Lawrence, 2015). This finding was not inflated by prior depletion condition (i.e., no differences in overall blast intensities between depleted and non-depleted participants). Whilst the validity studies of the Taylor paradigm have been conducted in Western countries (e.g., Giancola \& Parrott, 2008; Elson, 2016), our result indicates the possibility that the CRTT may be less sensitive to activate differential levels of aggression in the Indonesian sample. These notable divergences need further exploration.

In the CRTT, the Indonesian participants might have simply complied with the instruction to win the task, and were paying less attention to the disguised provocation procedure (i.e., the noise blasts they received following each losing trial). As a result, they might spontaneously deliver blast levels at any random point. However, if this explanation holds, it is unclear why sex differences in the CRTT in Yusainy and Lawrence (2015) British sample also occurred in this sample. More plausible is that the Indonesian may be less sensitive to the provocation procedure in the CRTT, as the sample has strict sanctions against the use of direct aggression. Many critiques have been addressed with regards to the unstandardised use of the CRTT (Elson et al., 2014; for various quantification strategies of CRTT see Elson et al., 2014). Given the ethical and practical issues in aggression research, however, most of labbased aggression alternatives paradigms are limited to measuring aggression either indirectly or in the form of verbal aggression (McCarthy et al., 2018).

Crucially, the conditions for the mindfulness induction and depletion task were comparable across cultures. As found in the replicated study with British sample, the 15-min mindfulness breathing exercise increased mindfulness state of decentering, but not curiosity. Dovetail with past research demonstrating that depletion manipulations have no impact on mood (Hagger et al., 2010), our participants reported no changes in post-depletion mood. Further, they submitted higher ratings in difficulty and effort of the attention control task, indicating that this task was effective to induce depletion. Of note, participant's perceptions of difficulty and effort were used initially in Baumeister et al. (1998) test of depletion, and are now commonly employed as subjective check of depletion state (Hagger et al. 2010). Physiological check such as reductions in blood glucose was suggested elsewhere (e.g., Gailliot \& Baumeister, 2007), however evidential value of this manipulation check is currently weak (Vadillo, Gold, \& Osman, 2016).

Our main analysis showed that despite participants' lack of formal experience in mindfulness practices, mindfulness induction moderated the link between ego- 
depletion and blast intensity under low/moderate provocation. This finding reiterates the result from the British sample, suggesting that the short-term benefits of mindfulness in counteracting the effect of depleted self-control resource on direct aggression may operate similarly across cultures outside training of mindfulness. Additionally, under high provocation trials, the moderating effect of mindfulness on the depletion and blast intensity link diminished. This latter finding is in agreement with past research showing that a very intense level of provocation is commonly sufficient to decrease the effect of a number of variables, such as sex differences (Bettencourt \& Miller, 1996), and initially non-aggressive opponent's behaviour (Lawrence \& Hutchinson, 2014) on direct aggression (i.e., ceiling effect).

Controlling for participants' sex provided further support for the moderation model. As found in the British sample, being Indonesian male also increased the likelihood of higher levels of blast intensities on no provocation and low provocations, but the effect of sex diminished on high provocations. This finding is in line with a meta-analysis in the Western cultures (Bettencourt \& Miller, 1996). They concluded that for females, the differences between aggression under provocation and neutral conditions are expected to be larger than for males, indicating the importance of provocation as a moderator of sex differences in aggression. Thus justification to display aggressive behaviour that is normally held in check by sex role norms appears to work similarly for females across cultures.

Mirroring the effect of mindfulness induction on post-depletion aggression, depleted participants with mindfulness induction showed a better self-control performance (assessed by changes in handgrip duration relative to baseline) than depleted participants with no mindfulness induction when self-control performance was measured prior to the CRTT. There was also a usual main effect of depletion condition, and positive main effect of mindfulness induction on self-control performance. The main effect of mindfulness was also shown in the original study with British sample, but the moderation of mindfulness on the depletion and handgrip stamina link was not evident. In the context where individuals lack self-control resource, the benefit of mindfulness induction in fostering a higher resistance to depletion (see Friese, Messner, \& Schaffner, 2012) might have been stronger for our current sample.

While the main effect of depletion and mindfulness induction persisted when selfcontrol performance was measured after the experience of provocation in the CRTT, the moderation of mindfulness did not survive. At this point, the effect of mindfulness on self-control performance was no longer dependent upon individuals having been subjected to the ego-depletion condition. A similar result was obtained in the original study with British sample. It seems that the short-term moderation effect of brief mindfulness inductions would not hold up for an extended period of time in particular when people have experienced provocation. In addition, females demonstrated increased final self-control performance.

The benefit of mindfulness induction on the maximum blast latency following depletion was noticeable in particular for females. Specifically, depleted Indonesian females who received mindfulness induction were less likely to deliver the maximum blast earlier compared to depleted females with no mindfulness 
induction. Giancola and Parrott (2008) posit that shock intensity as an explicit and blunt form of aggression, whereas shock duration is an implicit, subtle, and less amenable to influences of social desirability and impression management. The seemingly ambiguous nature of the latency measure is arguably similar to shock duration, and thus perceived as more suitable measure for female aggression than for male aggression in the current sample due to its less overt aspect. Thus for females, mindfulness induction reduced direct aggression in both the explicit form (blast intensity) and the subtle/implicit form (blast latency); whereas for males, reductions in aggression due to mindfulness induction occurred mainly in the explicit form of aggression. Nevertheless, considerable caution must be exercised in interpreting the results due to the absence of the usual main effect of depletion for the model of maximum blast latency.

Apart from the argument that mindfulness should be more applicable in Indonesia given its origin in Eastern contemplative traditions, our central theoretical construct was the cultural differences. We used the cultural differences distinction based on the characteristics of general population, and did not measure participants' adherence to the Eastern culture directly. University student samples are subjected to the influences of modernisation. For the Indonesians, these influences may include a shift from traditional collectivism to contemporary individualism, resulting in smaller cross-cultural differences than they would be in the general population.

\section{Conclusion}

We provide exploration of Western's operationalisation of mindfulness, selfcontrol ego-depletion, and aggression in the context of an Eastern culture, specifically Indonesia. As in the replicated Yusainy and Lawrence (2015) experiment with British sample, our participants postdepletion aggression (i.e., blast intensity under low/moderate provocation) was reduced following a brief induction of mindfulness state. The benefits might have been stronger for the Indonesians since the moderation of mindfulness extended to performance in a second self-control task (i.e., pre-aggression handgrip stamina). For female participants, mindfulness induction was also beneficial to reduce the harmful impact of depletion on our additional measure of implicit aggression (i.e., maximum blast latency in the CRTT).

\section{Recommendation}

Further replication study is warranted with regards to the impact of mindfulness on the subtle, implicit form of aggressive behaviour. More research is also crucial on the efficacy of the CRTT as a method of provocation. A more comprehensive view of the dynamics between mindfulness, selfcontrol ego-depletion, and aggression across cultures requires direct measures of cultural values and consideration of the role of other constructs (e.g., religion, Ramirez, 2007).

\section{Acknowledgment}

We wish to thank Claire Lawrence for her mindful guidance. We are also indebted to Nur Arfa Efrilega and Yusnike Windy Rahayuningtyas for their assistance with data collection. 


\section{References}

Allen, J. J., Anderson, C. A., \& Bushman, B. J. (2018). The general aggression model. Current Opinion in Psychology, 19, 75-89. doi: 10.1016/j.copsyc.2017.03.034

Anderson, C. A., Buckley, K. E., \& Carnagey, N. L. (2008). Creating your own hostile environment: A laboratory examination of trait aggressiveness and the violence escalation and the violence escalation cycle. Personality and Social Psychology Bulletin, 34(4), 462-473. doi: 10.1177/0146167207311282

Anderson, C. A., \& Bushman, B. J. (2002). Human aggression. Annual Review of Psychology, 53(1), 27-51. doi: 10.1146/annurev.psych.53.100901.1352 31

Archer, J. (2004). Sex differences in aggression in real-world settings: A meta-analytic review. Review of General Psychology, 8(4), 291-322. doi: 10.1037/ 1089-2680.8.4.291

Baron, B. A., \& Richardson, D. R. (1994). Perspectives in social psychology: Human aggression (2nd ed.). New York: Plenum Press.

Bartholow, B. D. (2018). The aggressive brain: Insights from neuroscience. Current Opinion in Psychology, 19, 60-64. doi: 10.1016/j.copsyc.2017.04.002

Baumeister, R. F., Bratslavsky, E., Muraven, M., \& Tice, D. M. (1998). Ego depletion: Is the active self a limited resource? Journal of Personality and Social Psychology, 74, 1252-1265. doi: 10.1037/0022-3514.74.5.1252

Baumeister, R. F., Heatherton, T. F., \& Tice, D. M. (1994). Losing control: How and why people fail at self-regulation. San Diego, CA: Academic Press.
Baumeister, R. F., Vohs, K. D., \& Tice, D. M. (2007). The strength model of selfcontrol. Current Directions in Psychological Science, 16(6), 351-355. doi: 10.1111/j.1467-8721.2007.00534.x

Bergeron, N., \& Schneider, B. H. (2005). Explaining cross-national differences in peer-directed aggression: A quantitative synthesis. Aggressive Behavior, 31(2), 116-137. doi: 10.1002/ab.20049

Bergmüller, S. (2013). The relationship between cultural individualismcollectivism and student aggression across 62 countries. Aggressive Behavior, 39(3), 182-200. doi: 10.1002/ab.21472

Berkowitz, L. (1993). Aggression: Its causes, consequences, and control. New York: McGraw-Hill.

Bettencourt, B. A., \& Miller, N. (1996). Gender differences in aggression as a function of provocation: A metaanalysis. Psychological Bulletin, 119(3), 422-447. doi: 10.1037/0033-2909. 119.3.422

Bishop, S. R., Lau, M., Shapiro, S., Carlson, L., Anderson, N. D., Carmody, J., ... Devins, G. (2004). Mindfulness: A proposed operational definition. Clinical Psychology: Science and Practice, 11(3), 230-241. doi: 10.1093/clipsy.bph077

Björkqvist, K., Lagerspetz, K. M., \& Kaukianen, A. (1992). Do girls manipulate and boys fight? Developmental trends in regard to direct and indirect aggression. Aggressive Behavior, 18(2), 117-127. doi: 10.1002/10982337(1992)18:2<117::AIDAB2480180205>3.0.CO;2-3

Bushman, B. J. (2002). Does venting anger feed or extinguish the flame? Catharsis, rumination, distraction, anger, and aggressive responding. Personality and 
Social Psychology Bulletin, 28(6), 724731. doi: $10.1177 / 0146167202289002$

Caspi, A. (2000). The child is father of the man: Personality continuities from childhood to adulthood. Journal of Personality and Social Psychology, 78(1), 158-172. doi: 10.1037/0022-3514. 78.1.158

Crick, N. R., \& Dodge, K. A. (1996). Social information-processing mechanisms in reactive and proactive aggression. Child Development, 67, 993 - 1002. doi: 10.1111/j.1467-8624.1996.tb01778.x

Denson, T. F. (2013). The multiple systems model of angry rumination. Personality and Social Psychology Review, 17(2), 103123. doi: $10.1177 / 1088868312467086$

Denson, T. F., DeWall, C. N., \& Finkel, E. J. (2012). Self-control and aggression. Current Directions in Psychological Science, 21(1), 20-25. doi: $10.1177 / 0963721411429451$

Dickerson, S. S., \& Kemeny, M. E. (2004). Acute stressors and cortisol responses: a theoretical integration and synthesis of laboratory research. Psychological Bulletin, 130(3), 355-391. doi: 10.1037/ 0033-2909.130.3.355

Elson, M. (2016). FlexibleMeasures.com: Competitive reaction time task. doi: 10 . 17605/ OSF.IO/4G7FV

Elson, M., Mohseni, M. R., Breuer, J., Scharkow, M. \& Quandt, T. (2014). Press CRTT to measure aggressive behavior: The unstandardized use of the competitive reaction time task in aggression research. Psychological Assessment, 26, 419-432, doi: 10.1037/ a0035569

Finkel, E. J., DeWall, C. N., Slotter, E. B., Oaten, M., \& Foshee, V. A. (2009). Selfregulatory failure and intimate partner violence perpetration. Journal of
Personality and Social Psychology, 97(3), 483-499. doi: 10.1037/a0015433

French, D. C., Jansen, E. A., \& Pidada, S. (2002). United States and Indonesian children's and adolescents' reports of relational aggression by disliked peers. Child Development, 73(4), 1143-1150. doi: $10.1111 / 1467-8624.00463$

Friese, M., Messner, C., \& Schaffner, Y. (2012). Mindfulness meditation counteracts self-control depletion. Consciousness and Cognition, 21(2), 1016-1022. doi: 10.1016/ j.concog. 2012.01.00

Gailliot, M. T., \& Baumeister, R. F. (2007). The physiology of willpower: Linking blood glucose to self-control. Personality and Social Psychology Review, 11, 303-327. doi: $10.1177 / 0963721411429451$

Geen, R. G. (2001). Human aggression (2nd ed.). Buckingham, England: Open University Press.

Giancola, P. R., \& Parrott, D.J. (2008). Further evidence for the validity of the Taylor aggression paradigm. Aggressive Behavior, 34(2), 214-229. doi: 10.1002/ ab.20235

Gottfredson, M. R., \& Hirschi, T. (1990). A general theory of crime. Stanford, CA: Stanford University Press.

Hagger, M. S., Chatzisarantis, N.L.D., Alberts, H., Anggono, C.O., Batailler, C., Birt, A., Brand, R., Brandt, ... Zweinenberg, M. (2016). A multi-lab pre-registered replication of the egodepletion effect. Perspectives on Psychological Science, 11(4), 546-573. doi: $10.1177 / 1745691616652873$

Hagger, M. S., Wood, C., Chris, S., \& Chatzisarantis, N. L. D. (2010). Egodepletion and the strength model of self-control: A meta-analysis. 
Psychological Bulletin. 136(4), 495-525. doi: 10.1037/a0019486

Hayes, A. F. (2018). Introduction to mediation, moderation, and conditional process analysis: A regression-based approach ( $2^{\text {nd }}$ ed.). New York: Guilford.

Hofstede, G., Hofstede, G. J., \& Minkov, M. (2010). Cultures and organizations: Software of the mind (3rd ed.). New York: McGraw-Hill.

Howells, K., Tennant, A., Day, A., \& Elmer, R. (2010). Mindfulness in forensic mental health: Does it have a role? Mindfulness, 1(1), 4-9. doi: 10.1007/s12671-009-0001-x

Inzlicht, M., \& Gutsell, J. (2007). Running on empty: Neural signals for self-control failure. Psychological Science, 18(11), 933-937. doi: 10.1111/j.14679280.2007.02004.x

Ireland, J. L., \& Batool, S. (2018). Treatment for aggression: Promoting individual change. In J. L. Ireland, P.Birch, \& C. A. Ireland (Eds.), The Routledge international handbook of human aggression: Current issues and perspectives (pp. XX-XX). New York: Routledge.

Koentjoroningrat. (1985). Javanese culture. Singapore: Oxford University Press East Asia.

Lau, M. A., Bishop, S. R., Segal, Z. V., Buis, T., Anderson, N. D., Carlson, L., Shapiro, S., Carmody, J., Abbey, S., \& Devins, G. (2006). The Toronto Mindfulness Scale: Development and validation. Journal of Clinical Psychology, 62(12), 1445-1467.

doi: 10.1002/jclp.20326

Lawrence, C., \& Hutchinson, L. (2014). The impact of non-aggressive behaviour early in aggressive interactions: Sex differences in direct and indirect aggression in response to provocation.
British Journal of Psychology, 105, 127144. doi: 10.1111/bjop.12020

Markus, H. R., \& Kitayama, S. (1991). Culture and the self: Implications for cognition, emotion, and motivation. Psychological Review, 98(2), 224-253. doi: 10.1037/0033-295X.98.2.224

McCarthy, R. J., \& Elson, M. (2018). A conceptual review of lab-based aggression paradigms. Collabra: Psychology, 4(1), 4, 1-12. doi: 10.1525/collabra.104

McEllistrem, J. E. (2004). Affective and predatory violence: A bimodal classification system of human aggression and violence. Aggression and Violent Behavior, 10(1), 1-30. doi: 10. 10.1016/j.avb.2003.06.002

Moffitt, T., Arseneault, L., Belsky, D., Dickson, N., Hancox, R., Harrington, H. L., .. Caspi, A. (2011). A gradient of childhood self-control predicts health, wealth, and public safety. Proceedings of the National Academy of Sciences, 108(7), 2693-2698.

doi: 10.1073/pnas. 1010076108

Ramirez, J. M. (2007). Justification of aggression in several Asian and European countries with different religious and cultural background. International Journal of Behavioral Development, 31(51), 9-15. Retrieved from http://eprints.ucm.es/8417/

Seeley, E. A., \& Gardner, W. L. (2003). The "selfless" and self-regulation: The role of chronic other orientation in averting self-regulatory depletion. Self and Identity, 2(2), 103-117. doi: $10.1080 / 15298860309034$

Shonin, E., Gordon W. V., Slade, K., \& Griffiths M. D. (2013). Mindfulness and other Buddhist-derived interventions in correctional settings: A systematic review. Aggression and Violent Behavior, 


\section{POST-DEPLETION AGGRESSION RESTRAINED}

18(3), $\quad 365-372 . \quad$ doi: 10.1016/j.avb.2013.01.002.

Staller, M. S., Christiansen, P., Zaiser, B., Körner, S., \& Cole, J. C. (2017). Do they aggress earlier? Investigating the effects of ego depletion on police officers' use of force behavior. Journal of Police and Criminal Psychology, 1, 1-13. doi: 10.1007/s11896-017-9249-6

Stucke, T. S., \& Baumeister, R. F. (2006). Ego depletion and aggressive behavior: Is the inhibition of aggression a limited resource? European Journal of Social Psychology, 36(1), 1-13. doi: 10.1002/ejsp.285

Taylor, S. (1967). Aggressive behavior and physiological arousal as a function of provocation and the tendency to inhibit aggression. Journal of Personality, 35(2), 297-310. doi: 10.1111/j.14676494.1967.tb01430.x

Teper, R., Segal, Z., \& Inzlicht, M. (2013). Inside the mindful mind: How mindfulness enhances emotion regulation through improvements in executive control. Current Directions in Psychological Science, 22(6), 449-454. doi: $10.1177 / 0963721413495869$

Trimulyaningsih, N. (2017). Konsep kepribadian matang dalam budaya Jawa-Islam: Menjawab tantangan globalisasi. Buletin Psikologi, 25(2), 8998. doi:

10.22146/buletinpsikologi.28728

Vadillo, M. A., Gold, N., \& Osman, M. (2016). The bitter truth about sugar and willpower: The limited evidential value of the glucose model of ego depletion. Psychological Science, 27(9), 1207-1214. doi: 10.1177/0956797616654911

Van Dam, N. T., van Vugt, M. K., Vago, D. R., Schmalzl, L., Saron, C. D., Olendzki, A., . . Meyer, D. E. (2018). Mind the hype: A critical evaluation and prescriptive agenda for research on mindfulness and meditation. Perspectives on Psychological Science, 13(1) 36-61. doi: $10.1177 / 1745691617709589$

Wallace, A. B., \& Bodhi, B. (2006). The nature of mindfulness and its role in Buddhist meditation: A correspondence between B. Alan Wallace and the venerable Bhikkhu Bodhi. Unpublished manuscript. Santa Barbara, California: Santa Barbara Institute for Consciousness Studies.

Watson, D., Clark, L. A, Tellegen, A. (1988). Development and validation of brief measures of positive and negative affect: the PANAS scales. Journal of Personality and Social Psychology, 54(6), 1063-1070. doi: 10.1037/00223514.54.6.106

Williams, J. M. G., \& Penman, D. (2011). Mindfulness: An eight-week plan for finding peace in a frantic world (Track 1). Oxford: Piatkus.

Wrangman, R. W. (2017). Two types of aggression in human evolution. Proceedings of the National Academy of Sciences, 115(2), 245-253. doi: 10.1073/pnas. 1713611115

Yusainy, C. (2017). Feeling full or empty inside? Peran perbedaan individual dalam struktur pengalaman afektif. Jurnal Psikologi, 44(1), 1-17. doi: 10.22146/jpsi.18377

Yusainy, C. (2015). Quo vadis Psikologi sebagai sebuah kajian ilmiah? Buletin Psikologi, 23(1), 51-56. doi: 10.22146/bpsi.10577

Yusainy, C., \& Lawrence, C. (2015). Brief mindfulness induction could reduce aggression after depletion. Consciousness and Cognition, 33(1), 125134. doi: 10.1016/j.concog.2014.12.008 
Yusainy, C., \& Lawrence, C. (2014). 64(1), 78-83. doi: 10.1016/ Relating mindfulness and aggression j.paid.2014.02.015

to harm to the self and to others.

Personality and Individual Differences, 